\title{
Mesenteric blood flow is related to disease activity and risk of relapse in ulcerative colitis: a prospective follow up study
}

D Ludwig, S Wiener, A Brüning, K Schwarting, G Jantschek, K Fellermann, M Stahl, E F Stange

\begin{abstract}
Background-The diagnostic significance of increased splanchnic blood flow in ulcerative colitis is unclear. This prospective study was therefore undertaken to define the role of Doppler sonography in the assessment of disease activity and in the prediction of early relapse.

Subjects/Methods-Splanchnic flowmetry was performed in 76 patients with ulcerative colitis (47 with active disease and 29 in remission), six with infectious colitis, and 13 healthy controls during fasting and 30 minutes after ingestion of a standardised meal. Twenty seven of the patients with ulcerative colitis and all patients with infectious colitis were investigated during the active state as well as in clinical remission and followed up for six months. Flow velocity and pulsatility index (PI) of the superior (SMA) and inferior (IMA) mesenteric arteries and the portal vein were related to clinical (Truelove index), laboratory (C-reactive protein), and endoscopic (Sutherland index) parameters of disease activity.
\end{abstract}

Results-The mean flow velocity of the IMA correlated closest with clinical activity (Truelove, $r=0.41, p<0.005$ ), the PI with $\mathrm{C}$-reactive protein $(r=0.30, \mathrm{p}<0.05)$, and endoscopic activity $(r=0.45$, $\mathbf{p}<0.001)$. All patients in remission after six months $(14 / 14)$ or with infectious colitis (6/6) showed an increase in PI of the IMA compared with the initial measurement during active disease (mean increase for ulcerative colitis $+36 \%$ and for infectious colitis $+77 \%$ ). In contrast, most patients with later relapse or surgery (11/13) had decreased PI during initial remission (mean decrease $-12 \%$ ). The positive predictive value of this index for maintenance of remission was 0.77. Flow variables of the SMA and portal vein displayed weaker correlations.

Conclusions-Flow measurements in the IMA are closely related to clinical and endoscopic disease activity in patients with ulcerative colitis. Repeated measurement of the PI allows estimation of the risk of recurrence.

(Gut 1999;45:546-552)

Keywords: Doppler ultrasound; splanchnic haemodynamics; disease activity; ulcerative colitis; infectious colitis; inflammatory bowel disease
In ulcerative colitis, assessment of disease activity and prediction of relapse are of major clinical importance. The Truelove and Witts criteria $^{1}$ are well established for estimating the overall clinical status. Severity of inflammatory activity is better assessed using laboratory variables such as C-reactive protein. ${ }^{2}$ For patients with mild disease, histological appearance of the colonic mucosa is more reliable and of prognostic value. $^{3}$

Echo-Doppler flowmetry allows noninvasive access to the splanchnic circulation and permits the measurement of various variables such as flow velocity and flow volume in mesenteric arterial and venous vasculature. Although the precision of absolute measurements of flow rate is probably limited, estimation of short term changes in flow is quite reliable, ${ }^{45}$ even if a relevant interobserver variability is considered. ${ }^{6}{ }^{7}$ Analysis of postprandial hyperaemia has been found to be superior to baseline measurements in the assessment of postprandial abdominal pain ${ }^{8}$ and portal hypertension. ${ }^{9}$ Doppler flowmetry in inflammatory bowel disease has been conducted in a restricted number of fasting patients with analysis limited to the portal vein (PV) and superior mesenteric artery (SMA). ${ }^{10}$

This prospective evaluation was therefore performed to relate splanchnic haemodynamics, including those of the inferior mesenteric artery (IMA), to the severity and extent of ulcerative colitis using Echo-Doppler measurements in both the preprandial and postprandial state. In addition, we followed patients with repeated measurements in active disease and during remission to determine whether this method would be of predictive value with respect to early relapse.

\section{Materials and methods}

HEALTHY CONTROLS AND PATIENTS

Thirteen healthy subjects (mean age 27 (range 22-31) years) were studied in order to define the physiological preprandial and postprandial mesenteric and portal haemodynamics under control conditions (table 1). Between September 1995 and March 1998, 85 consecutive patients (mean age 36 (range 18-69) years) of varying disease severity were included in the study. Seventy six patients were evaluated; nine

Abbreviations used in this paper: HA, hepatic artery; IMA, inferior mesenteric artery; PI, pulsatility index; PV, portal vein; SMA, superior mesenteric artery; $\mathrm{V}_{\min }$, end diastolic velocity; $\mathrm{V}_{\max }$, peak systolic velocity; $\mathrm{V}_{\text {mean }}$, mean velocity per unit time. 
Table 1 Patient characteristics at entry

\begin{tabular}{lll}
\hline & $\begin{array}{l}\text { Controls } \\
(n=13)\end{array}$ & $\begin{array}{l}\text { Ulcerative colitis } \\
(n=76)\end{array}$ \\
\hline Sex $(\mathrm{M} / \mathrm{F})$ & $7 / 6$ & $45 / 31$ \\
Age (years) & $27(3)(22-31)$ & $36(15)(18-69)$ \\
Height (cm) & $176(9)$ & $180(8)$ \\
Weight (kg) & $71(9)$ & $69(9)$ \\
Truelove class (n) & & \\
$\quad$ I & - & 29 \\
II & - & 21 \\
$\quad$ III & - & 26 \\
Disease extent (n) & & \\
$\quad$ Extensive colitis & - & 28 \\
$\quad$ Left-sided colitis $\dagger$ & - & 48 \\
\hline
\end{tabular}

Values are expressed as mean (SD) (range).

^Including patients with total and subtotal colitis; tincluding patients with proctosigmoiditis, but not isolated proctitis.

had to be excluded because of unsatisfactory visualisation of the SMA $(n=2)$ or IMA ( $n=$ 6 ) in the preprandial or postprandial state or inability to drink the liquid test meal as required $(n=1)$. Failure of visualisation of the hepatic artery (HA) $(n=17)$ or portal vein (PV) $(\mathrm{n}=2)$ was not an exclusion criterion. The diagnosis, activity, and disease extension of ulcerative colitis was established on the basis of typical symptomatic, radiographic, and endoscopic criteria. Confirmation of the diagnosis and disease extent was provided by colonoscopy or proctosigmoidoscopy with biopsy. ${ }^{12}$ Doppler examination was performed within the previous or following three days of endoscopy. Infectious colitis was excluded by negative stool culture for enteric pathogens.

Forty seven patients had active disease (Truelove class II and III), and 29 were in remission. Twenty eight patients had an extensive colitis (subtotal or total), and, in 48, inflammatory activity was limited to the left colon. Patients with isolated proctitis were not included.

Twenty seven patients assessed by Doppler sonography during the acute attack (Truelove class II or above) were followed up, and measurements were repeated when they were reaching clinical remission after $17 \pm 5$ days (range 8-31). At each time point, patients were evaluated with respect to clinical activity, endoscopic activity, and laboratory findings. Six months after the acute attack, all patients included in the follow up were again reassessed with respect to the course of the disease. The outcome at this time point was used for further comparative analysis.
For comparison, six patients with acute infectious colitis (Campylobacter jejuni $(\mathrm{n}=2)$, Clostridium difficile $(\mathrm{n}=2)$, Salmonella typhimurium $(\mathrm{n}=1)$, no agent isolated $(\mathrm{n}=1)$ ) were analysed, and follow up measurements were performed after complete clinical remission (no diarrhoea, C-reactive protein $<5 \mathrm{mg} / \mathrm{dl}$ ).

EQUIPMENT AND INVESTIGATIONAL TECHNIQUES Laboratory assays were performed using standard procedures. Sonographic examination was made at rest in both normal controls and patients in the supine position after an overnight fast. The computerised instrument (Acuson 128; Acuson Corporation, Mountain View, California, USA) was equipped with a real time B mode sector scanner (3.5 MHz) and integrated pulsed Doppler flowmeter (4 $\mathrm{MHz}$ ). A cut off filter of $100 \mathrm{~Hz}$ was installed to exclude possible artefacts from vessel wall motion. Flow direction was determined by colour coded imaging, and velocity was calculated from the spectral shift of the Doppler signal (peak systolic velocity $\left(\mathrm{V}_{\max }\right)$ and end diastolic velocity $\left.\left(\mathrm{V}_{\min }\right)\right)$. The time averaged velocity (mean velocity per unit time $\left(\mathrm{V}_{\text {mean }}\right)$ ) is a computer derived value calculated by integrating the area under each velocity waveform. For arterial vessels, the pulsatility index (PI) was obtained by Doppler waveform according to the formula: $\mathrm{PI}=\left(\mathrm{V}_{\max }-\mathrm{V}_{\min }\right) / \mathrm{V}_{\text {mean }}{ }^{13}$ The PI is related to the total resistance to blood flow, including resistance, compliance, and congestion of the respective vascular bed. ${ }^{14}$ Each measurement was taken in triplicate during quiet respiration and averaged during a three second interval.

All vessels were examined in a consecutive fashion in a predetermined order (V1-V4). SMA (V1) and IMA (V2) were both identified at their origin and followed longitudinally to the more distal straight parts $(3-5 \mathrm{~cm}$ from the origin). PV (V3) and HA (V4) were always measured on the crossing point of both vessels, which is a definite position reducing variability. ${ }^{5}$ For all vessels, the transducer was positioned in each case to obtain a maximum Doppler signal and to keep the angle of insonation below $60^{\circ}$. As considerable variation in Doppler flow velocity may result from changes in the angle of insonation, ${ }^{5}$ measurements of fasting and postprandial flow velocity

Table 2 Doppler ultrasound measurements of splanchnic haemodynamics in patients with active and inactive ulcerative colitis and healthy controls

\begin{tabular}{|c|c|c|c|c|c|c|}
\hline & \multicolumn{2}{|c|}{$S M A\left(V_{\text {mean }}, \mathrm{cm} / \mathrm{s}\right)$} & \multicolumn{2}{|c|}{$I M A\left(V_{\text {mean }}, \mathrm{cm} / \mathrm{s}\right)$} & \multicolumn{2}{|c|}{$P V\left(V_{\text {mean }}, \mathrm{cm} / \mathrm{s}\right)$} \\
\hline & Baseline & Postprandial & Baseline & Postprandial & Baseline & Postprandial \\
\hline Controls $(n=13)$ & $28(7)$ & $47(18)$ & $15(3)$ & $19(5)$ & $18(3)$ & $23(3)$ \\
\hline \multicolumn{7}{|l|}{ Ulcerative colitis } \\
\hline Remission $(n=29)$ & $40(13)^{\star}$ & $85(30)^{\star}$ & $27(9)^{\star}$ & $32(15)^{\star}$ & $20(7)$ & $30(7)$ \\
\hline Moderate activity $(n=21)$ & $50(19)^{\star} \dagger$ & $109(31)^{\star} \dagger$ & $36(12) \star \mathbb{S}$ & $42(10)^{\star} \dagger$ & $29(15)$ & $36(12)$ \\
\hline Severe activity $(n=26)$ & $51(21)^{\star} \dagger$ & $106(34)^{\star} t$ & $45(13)^{\star} \ddagger$ & $48(20)^{\star} \dagger$ & $39(22)$ & $46(15) \Phi$ \\
\hline
\end{tabular}

Values are expressed as means (SD). Disease activity was graded following the Truelove index. All values for superior mesenteric artery (SMA) and inferior mesenteric artery (IMA) were significantly higher in patients with ulcerative colitis than in healthy controls ( ${ }^{\mathrm{S}} \mathrm{S}$ A $\mathrm{p}<0.05$, IMA $\left.\mathrm{p}<0.005\right)$. Patients with severe and moderate activity had higher fasting and postprandial values for SMA and IMA- $\mathrm{V}_{\text {mean }}$ than patients in remission $(\dagger \mathrm{p}<0.05)$. Fasting IMA- $\mathrm{V}_{\text {mean }}$ increased gradually with disease activity $(\neq \mathrm{p}<0.05 \mathrm{severe} v$ moderate, $\mathrm{p}<0.001$ severe $v$ remission; $\$ \mathrm{p}<0.01$ moderate $v$ remission). Mean portal flow velocity was significantly increased in patients with severe disease activity during fasting $(\uparrow \mathrm{p}<0.05 v$ remission), but especially in the postprandial state $(\mathrm{p}<0.01 v$ remission and moderate activity).

$\mathrm{PV}$, portal vein; $\mathrm{V}_{\text {mean }}$, mean velocity per unit time. 

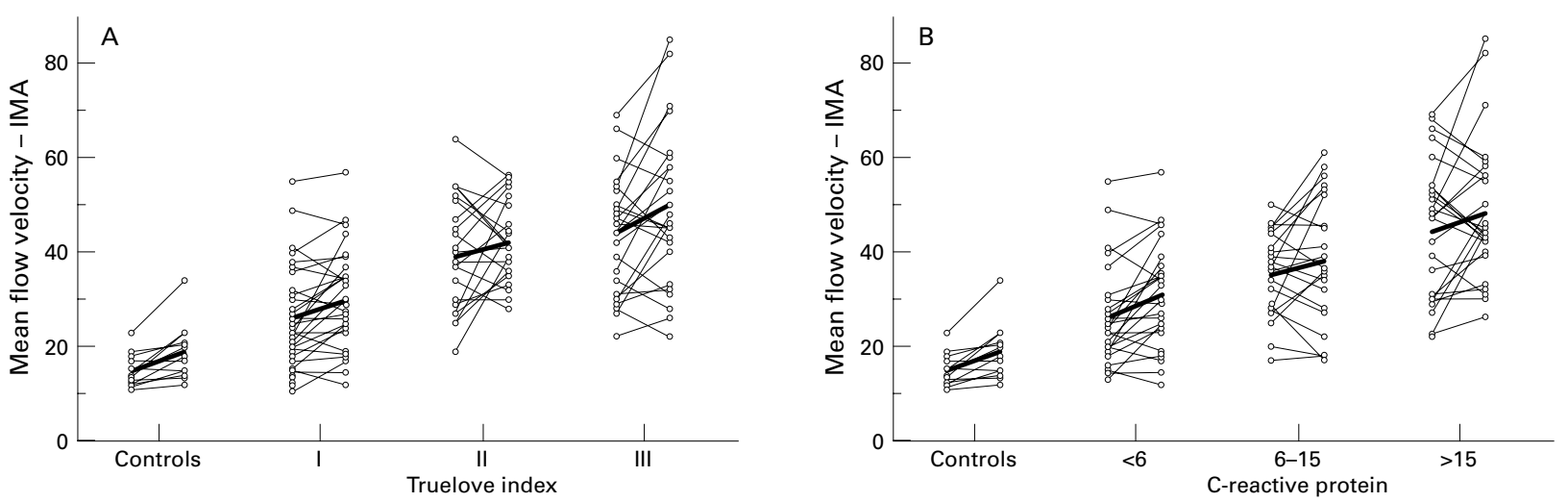

Figure 1 Effect of a standard meal on mean flow velocity in the inferior mesenteric artery (IMA) in controls and patients with ulcerative colitis. Mean baseline and mean postprandial values for each patient group are indicated by a heavy solid line. Baseline and postprandial mean flow velocity increased gradually with disease activity (assessed with the Truelove index (A) and C-reactive protein (B)).

variables were taken at the same point approximating the same angle $\left( \pm 5^{\circ}\right)$ in each of the controls and patients studied. In the patients re-evaluated after clinical remission, ultrasonographic pictures taken during a previous examination were used to reproduce the same section, sample volume, and angle of insonation. All measurements were taken at baseline within a 10 minute interval and repeated 30 minutes after ingestion of a standardised liquid meal within the same time frame. The liquid meal (Fresubin; Fresenius, Bad Homburg, Germany) was given within a 10 minute period at a dose of $5 \mathrm{ml} / \mathrm{kg}$ body weight and contained $3.8 \mathrm{~g}$ protein, $3.4 \mathrm{~g}$ fat, $13.8 \mathrm{~g}$ carbohydrate, and $418 \mathrm{~kJ}$ per $100 \mathrm{ml}$.

To minimise the variability of ultrasound measurements, all investigations were performed by a single investigator (DL). The examiner was not blinded with respect to patient history, but was unaware of the actual clinical, endoscopic, and laboratory activity state. Intraobserver variability has been tested previously in 12 healthy volunteers on four consecutive days. The coefficients of variation for baseline and postprandial mean velocity and PI within the same day were $8,9,6$, and $6 \%$ for SMA and $10,12,8$, and $9 \%$ for IMA respectively. Variability on consecutive days for the same variables was $10,14,8$, and $9 \%$ for SMA and $12,15,9$, and $9 \%$ for IMA measurements. Mean portal flow velocity varied between 10 and $13 \%$ for preprandial and postprandial measurements on the same day and 10 and $14 \%$ for repeated measurements on consecutive days. Informed consent was obtained from each volunteer and patient. The ethical guidelines of the 1975 Declaration of Helsinki were followed throughout.

STATISTICAL ANALYSIS

The results are given for each group as mean (SD). Values with two variables were tested for significance with the Mann-Whitney U/Wilcoxon rank sum test. For data sets with more than two variables, a non-parametric analysis of variance (Kruskal Wallis one and two way analysis of variance) was performed. The best cut off point in the prediction of patient outcome was selected using the receiver operating characteristic curve. ${ }^{15} \mathrm{p}<0.05$ was considered significant (SPSS 6.1 for Windows Update; SPSS Inc, Chicago, Illinois, USA).

\section{Results}

\section{MESENTERIC HAEMODYNAMICS}

Preprandial and postprandial measurements of all vessels were obtained in controls. In patients, complete measurements of SMA, IMA, PV, and HA were possible in 98, 92, 98, and $80 \%$ respectively. Further evaluation was based on patients with complete preprandial and postprandial measurements of SMA and IMA ( $\mathrm{n}=76)$. In controls and patients, the mean flow velocity of the SMA (SMA- $\left.\mathrm{V}_{\text {mean }}\right)$ and PV (PV-V $\left.\mathrm{V}_{\text {mean }}\right)$ increased 30 minutes after the liquid meal (range of the means $67-122 \%$ and $28-53 \%$ respectively), whereas flow measurements in the IMA (IMA- $\mathrm{V}_{\text {mean }}$ ) varied little between the preprandial and postprandial state in both groups (table 2).

In all groups, $S M A-V_{\text {mean }}$ and IMA- $V_{\text {mean }}$ were higher at baseline and postprandially than in normal subjects $\quad(\mathrm{p}<0.05$ and $\mathrm{p}<0.005$

Table 3 Pulsatility index of splanchnic arteries in patients with active and inactive ulcerative colitis and healthy controls

\begin{tabular}{|c|c|c|c|c|c|c|}
\hline & \multicolumn{6}{|c|}{ Pulsatility index } \\
\hline & \multicolumn{2}{|l|}{$S M A$} & \multicolumn{2}{|l|}{$I M A$} & \multicolumn{2}{|l|}{$H A$} \\
\hline & Baseline & Postprandial & Baseline & Postprandial & Baseline & Postprandial \\
\hline Controls $(n=13)$ & $3.6(0.9)$ & $2.7(0.8)$ & $4.6(0.9)$ & $4.2(1.0)$ & $1.0(0.3)$ & $1.6(0.5)$ \\
\hline \multicolumn{7}{|l|}{ Ulcerative colitis } \\
\hline Remission $(n=29)$ & $3.1(0.7)$ & $1.8(0.5)^{\star}$ & $4.0(1.1)$ & $3.9(1.3)$ & $1.1(0.6)$ & $2.1(1.5)$ \\
\hline Moderate activity $(n=21)$ & $2.8(0.7)$ & $1.5(0.4)^{\star}$ & $3.4(0.9)^{\star}$ & $3.4(1.1)$ & $1.1(0.5)$ & $2.9(1.7)$ \\
\hline Severe activity $(n=26)$ & $2.9(0.9)$ & $1.7(0.5)^{\star}$ & $3.2(0.8) \dagger$ & $3.1(0.8)^{\star}$ & $1.1(0.4)$ & $2.2(1.1)$ \\
\hline
\end{tabular}

Values are expressed as means (SD). Disease activity was graded using the Truelove index (class I-III). *The pulsatility index of patients with ulcerative colitis was significantly different from controls $(\mathrm{p}<0.05)$. + In patients with severe disease activity the fasting pulsatility index was significantly lower than in controls $(p<0.001)$ and patients in clinical remission $(p<0.001)$.

SMA, superior mesenteric artery; IMA, inferior mesenteric artery; HA, hepatic artery. 


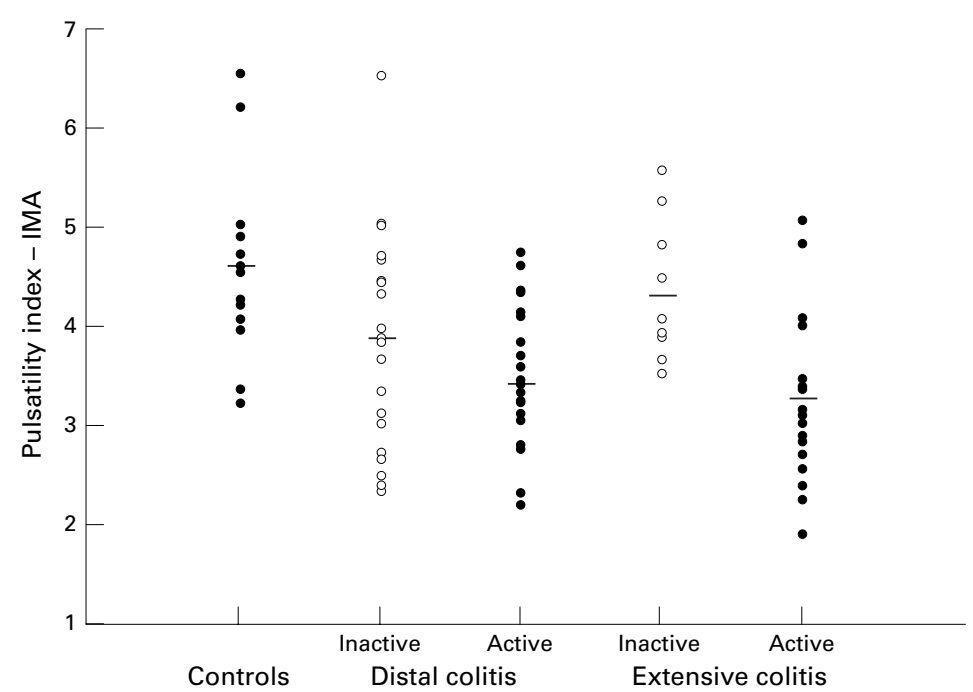

Figure 2 Fasting pulsatility index of the inferior mesenteric artery (IMA) in relation to localization and activity of disease in patients with ulcerative colitis compared with controls. Significant differences were obtained between patients with active and inactive extensive colitis and between all patients with active disease and controls. Mean values are given by a horizontal solid bar.
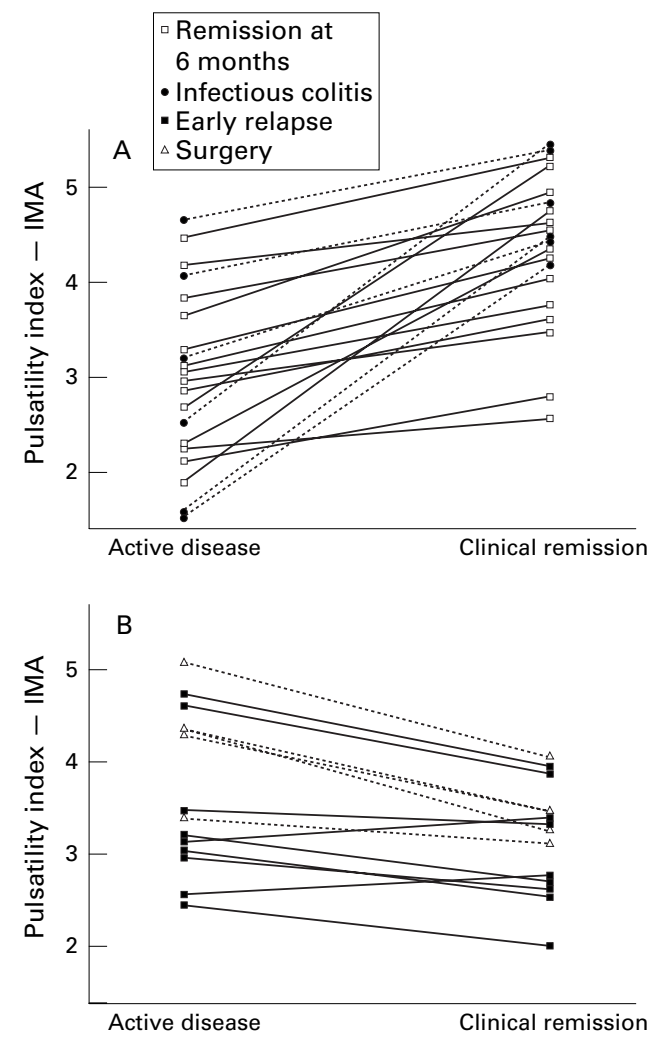

Figure 3 Fasting pulsatility index of the inferior mesenteric artery (IMA) measured in the active state and after clinical remission. All patients with sustained remission at six months and with infectious colitis $(A)$ showed an increase in pulsatility index, whereas most of those with early relapse or surgery (B) had decreased values despite clinical remission.

protein) and endoscopic disease activity were best related to the fasting PI of IMA $(r=0.30$, $\mathrm{p}<0.05$ and $r=0.45, \mathrm{p}<0.001$ respectively).

The PI of the SMA and IMA decreased gradually with disease activity, but correlated less well with the Truelove index than the $\mathrm{V}_{\text {mean }}$ (table 3). HA PI was not different from controls, but there was a tendency towards a greater increase after the meal in patients with ulcerative colitis (table 3 ).

C-reactive protein largely showed no relation to any flow variable of SMA, despite a trend towards increased postprandial values in patients with high inflammatory activity (Creactive protein $>15$; table 3 ). The fasting $\mathrm{V}_{\text {mean }}$ and even more so the PI of the IMA were closely related to concentration of C-reactive protein (fig 1B, table 3 ).

With respect to endoscopic disease activity, the fasting PI of the IMA was significantly lower in patients with active colitis $(3.4(0.7))$ than in patients in remission (4.1 (0.9); $\mathrm{p}<0.001)$ and controls (4.6 (0.9); $\mathrm{p}<0.001)$. This difference was also significant in the subgroup of patients with extensive colitis $(p<0.005)$, but not in patients with left sided colitis $(p=0.07)$ (fig 2$)$. Flow in the SMA, PV, and HA essentially showed no relation to disease extension and activity (data not shown).

The closest correlation between haemodynamic measurements and clinical disease activity was found for fasting $\mathrm{V}_{\text {mean }}$ of the IMA $(r=0.41, \mathrm{p}<0.005)$. The biological (C-reactive
PREDICTION OF RELAPSE

All measurements in patients of the follow up group were taken at least twice, with one measurement at admission and a second when reaching clinical remission. All patients reached clinical remission (Truelove class I) with standard treatment (oral prednisolone 1 $\mathrm{mg} / \mathrm{kg}$ body weight, tapered at $10 \mathrm{mg} /$ week; mesalamine $4 \mathrm{~g}$ enemas twice a day in left sided colitis). Maintenance treatment consisted of 5-aminosalicylic acid (mesalamine or olsalazine) 3-4 g/day in all patients.

At six months, 14 patients were still in complete remission (group I), whereas 13 had relapsed early $(n=9)$, or had to be operated on for bleeding $(n=1)$, or developed fulminant colitis refractory to medical treatment $(n=3)$ (group II). The two subgroups were comparable with respect to disease history, activity, and endoscopic parameters (table 4). Moreover the fasting PI values of the SMA and IMA during active disease were not different (table 4).

All patients with ulcerative colitis still in remission at six months and all patients with acute infectious colitis (group III) showed an increase in the fasting PI of the IMA during the second series of measurements - that is, after achieving remission (fig $3 \mathrm{~A}$ ). In contrast, nearly 
Table 4 Follow up patient characteristics at entry

\begin{tabular}{llll}
\hline & $\begin{array}{l}\text { Patients in remission } \\
(n=13)\end{array}$ & $\begin{array}{l}\text { Patients with } \\
\text { relapse }(n=14)\end{array}$ & $\begin{array}{l}\text { Infectious } \\
\text { colitis }(n=6)\end{array}$ \\
\hline Sex (M/F) & $4 / 9$ & $7 / 6$ & $5 / 1$ \\
Age (years) & $27(8)$ & $38(18)$ & $41(15)$ \\
Last previous attack (months) & $23(14)(0-62)$ & $24(23)(0-120)$ & - \\
$\begin{array}{l}\text { Duration of disease (months) } \\
\text { Disease activity (Rachmilewitz index) }\end{array}$ & $47(41)(0-122)$ & $75(55)(0-140)$ & - \\
$\begin{array}{l}\text { Disease extent (n) } \\
\quad \text { Extensive colitis }\end{array}$ & $9 / 13$ & $10(4)$ & - \\
$\quad$ Left-sided colitis & $4 / 13$ & $10 / 14$ & $6 / 6$ \\
$\begin{array}{l}\text { Pulsatility index } \\
\quad \text { SMA baseline }\end{array}$ & $2.7(0.6)$ & $4 / 14$ & - \\
$\quad$ IMA baseline & $3.3(0.8)$ & $2.9(0.4)$ & $3.6(1.2)$ \\
& & $3.7(1.0)$ & $2.9(1.4)$ \\
\hline
\end{tabular}

Values are expressed as means (SD) (range).

*Including patients with total and subtotal colitis; fincluding patients with proctosigmoiditis, but not proctitis; $\neq$ fasting pulsatility index values are given.

SMA, superior mesenteric artery; IMA, inferior mesenteric artery.

Table 5 Sensitivity, specificity and predictive values for difference of inferior mesenteric artery pulsatility index (PI) between the first (active state of disease) and the second measurement (remission) in the prediction of early relapse or persistent remission within six months

\begin{tabular}{llllll}
\hline $\begin{array}{l}\text { PI } \\
\text { increase } \\
\text { (remission) }\end{array}$ & $\begin{array}{l}\text { PI } \\
\text { decrease } \\
\text { (relapse) }\end{array}$ & Sensitivity & Specificity & $P P V$ & NPV \\
\hline 0.5 & 0.3 & 0.73 & 0.75 & 0.77 & 0.69 \\
0.7 & 0.4 & 0.66 & 0.66 & 0.65 & 0.54 \\
0.9 & 0.5 & 0.46 & 0.42 & 0.39 & 0.45 \\
\hline
\end{tabular}

$\mathrm{PPV}$, positive predictive value; NPV, negative predictive value.

all patients with early relapse or surgery $(11 / 13)$ had a lower PI in comparison with initial measurements (fig 3B). The absolute values of the PI during the second series of measurements were also significantly different (group I: 4.2 (0.4); group II: $3.1(0.6), \mathrm{p}<0.005$ $v$ group I; group III: $5.1(0.9), \mathrm{p}<0.001 v$ group I). There was no difference between patients in remission and patients with infectious colitis at this time point $(p>0.05)$. The receiver operating characteristic curve showed that the best cut off point in the prediction of patient outcome was an increase in PI of 0.5 for remission and a decrease in PI of 0.3 for early relapse. Positive and negative predictive values of IMA PI were 77 and $69 \%$ respectively (table 5).

The PI of SMA in all patients with ulcerative colitis was not related to patient outcome at six months in either the fasting or postprandial state (fig $4 \mathrm{~A}, \mathrm{~B}$ ). Considering only patients with extensive colitis, the PI increased in most nonrelapsed patients with ulcerative colitis (six of nine) and all six patients with infectious colitis. Most patients with extensive colitis and early recurrence of disease or surgery (eight of ten) showed decreased values when reassessed during remission.

\section{Discussion}

Hyperdynamic splanchnic blood flow in inflammatory bowel disease has been shown by different methods. Early angiographic and histopathological studies showed increased vascularity with increased mucosal to submucosal but reduced muscularis blood flow in active disease, which tended to decrease further in the late fibrosing stage. ${ }^{16}{ }^{17}$ However, there is no evidence for a pathogenetic sequence of events with primary vascular injury and consequent multifocal gastrointestinal infarction, as has
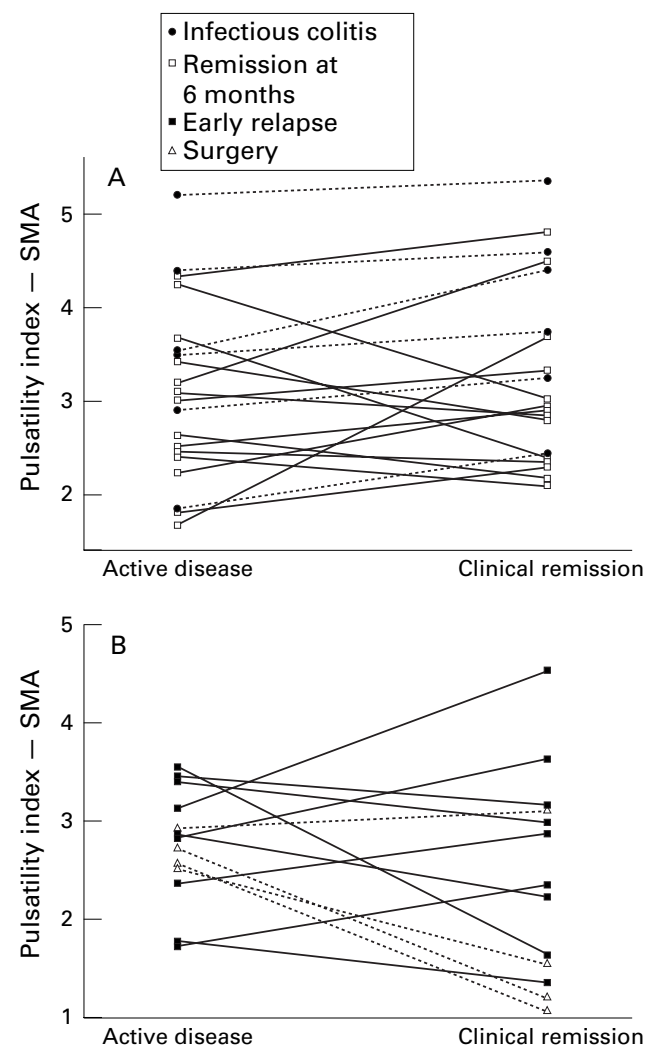

Figure 4 Fasting pulsatility index of the superior mesenteric artery (SMA) measured in the active state and after clinical remission. All patients with infectious colitis showed a moderate increase in pulsatility index $(A)$, whereas in ulcerative colitis there was no clear difference in patients with sustained remission at six months, early relapse, or surgery $(A+B)$.

been postulated for Crohn's disease. ${ }^{18}{ }^{19} \mathrm{~A}$ twoto six-fold increase in colonic blood flow has been recognised in inflammatory bowel diseases and experimental colitis. ${ }^{16}{ }^{20}$ Several mechanisms have been proposed to explain the colitis induced intestinal hyperaemia. These include an increase in mucosal and submucosal blood flow as the result of increased levels of arachidonic acid metabolites (prostaglandin $\left.\mathrm{E}_{2}\right)^{21}$ and other vasoactive agents (histamine, nitric oxide). ${ }^{22}{ }^{23}$ Expression of nitric oxide, a key messenger molecule directly involved in the mucosal inflammatory response and also the regulation of vasomotor tone, is enhanced in active ulcerative colitis. ${ }^{23-25}$ It remains unclear whether the tissue concentrations of these agents rise sufficiently during inflammation to cause vasodilatation. On the other hand, rectal blood flow measured by laser Doppler flowmetry has been found to be reduced in inactive but also active proctitis. ${ }^{26}$ The latter finding strongly favours the theory of increased arteriovenous shunting, but no direct measurements are available for the colon so far.

Doppler sonography is a suitable method for the non-invasive investigation of splanchnic haemodynamics. Previous studies have shown it to be accurate and reproducible in various pathophysiological conditions such as intestinal angina, ${ }^{8}$ portal hypertension, ${ }^{9}$ and inflammatory bowel diseases. ${ }^{10}{ }^{11}$ However, in previous studies, the patient groups were small. 
Measurements were performed only in the fasting state and IMA haemodynamics were not investigated. In addition, studies were not prospectively stratified according to clinical disease activity or extension and no follow up investigations were conducted with respect to long term remission versus early relapse. Thus this investigation is the first to relate prospectively fasting and postprandial splanchnic blood flow velocity to various activity variables and to relapse rate in patients with ulcerative colitis.

Technical and systematic problems of flow quantification are major sources of error: incorrect positioning of the Doppler sample volume within the vessel, difficulty in obtaining an optimal angle of insonation, and imprecise measurements of the lumen diameter all may lead to an overestimation or underestimation of flow of up to $24 \% .{ }^{57}$ Therefore we measured each vessel in a straight course of at least $3 \mathrm{~cm}$, excluded beam/vessel angles of more than $60^{\circ}$, and did not quantify blood flow volume. Our intraindividual variance ranging between 8 and $15 \%$ for mean velocity and 8 and $9 \%$ for PI measurements is acceptable and comparable with previous work. ${ }^{6}$ Even though measurements of mesenteric flow velocity have been considered less reliable than analysis of resistive and pulsatility index, ${ }^{1127}$ we found good reproducibility in follow up investigations, provided that interfering factors (ultrasound section, sample volume, insonation angle) were kept constant in a given subject. These findings agree with previous reports that the nonsystematic variance component is the key problem, suggesting the use of intraindividual flow measurements. ${ }^{5}$

As left sided and distal colitis would escape detection by measuring the SMA only, we evaluated blood flow in the IMA as well. Measurements in this vessel have been considered unreliable because of the small diameter or inconstant visualisation because of bowel gas. ${ }^{11}$ With considerable expertise, however, it is possible to obtain flow measurements in $93 \%$ of patients with a similar reproducibility to that for SMA.

Our results are generally consistent with the previously shown circulatory changes in splanchnic vessels in ulcerative colitis. ${ }^{10}{ }^{11}$ Like others, we observed an increase in fasting, but also postprandial blood flow of the PV in patients with severe disease activity. In contrast, we were not able to differentiate between active and quiescent ulcerative colitis by flow measurements in the SMA or PV. This difference may be due to the higher number of patients evaluated or a more precise definition of the level of activity and extent of disease in this study. Not previously assessed by others, we found a close correlation of flow variables of the IMA with clinical and also inflammatory disease activity in ulcerative colitis. The importance of the IMA is due to the fact that isolated left sided colitis and distally active pancolitis are found in most patients with ulcerative colitis.

To evaluate further the role of postprandial hyperaemia in patients with ulcerative colitis, we repeated all measurements 30 minutes postprandially, as it has been shown previously that the increase in intestinal hyperaemia peaks between 30 and 90 minutes after a mixed liquid meal. ${ }^{28}$ In animal and human studies, this postprandial increase in splanchnic blood flow has been found to be related to arteriolar vasodilatation in the mucosal and submucosal vessels of the small intestine, ${ }^{29}{ }^{30}$ combined with a reduced post-sinusoidal resistance ${ }^{29}$ and an increase in cardiac output. ${ }^{31}$ The underlying mechanism of this hyperaemia is also largely unknown, but cholinergic pathways are more likely than humoral factors to be involved in the control of postprandial mesenteric blood flow. ${ }^{28}$ Interestingly, the postprandial hyperaemia is not reduced in patients with ulcerative colitis. This finding is consistent with a normal absorptive capacity of the small bowel even in a state of high systemic inflammation as in severe colitis. Nonetheless, postprandial flow measurements appear to be dispensable because correlations with disease activity have been found that were comparable with baseline measurements.

Disease extent and activity assessed by endoscopy were also related to fasting flow velocity and PI of IMA. These results agree with previous comparative studies which found the correlation between clinical score systems such as Truelove and Witts score and endoscopic scoring systems to be satisfactory. ${ }^{32}$

The most important finding of this study with considerable clinical relevance was the relation between splanchnic blood flow measurements and early relapse of disease. Failure of the PI of IMA to increase when clinical remission was reached was highly indicative of an early relapse or surgery for complications. In contrast, patients with increased PI values remained in remission for at least six months. Measurements in the SMA were less reliable, but its analysis may be useful in patients with pancolitis, in whom analysis of the IMA may not be possible for technical reasons-for example, bowel gas.

These findings are relevant because it remains difficult to establish clinical, biological, or endoscopic variables that predict the response to steroid treatment or clinical relapse..$^{25} 33$ Compared with previous studies, ${ }^{34}{ }^{35}$ the general relapse rate within six months was rather high in our patients. This may be due to patient selection, as younger age ( $<50$ years) and extensive colitis are associated with a higher relapse rate and risk for surgery. ${ }^{34} 35$

The observed low PI in relapsing patients may be a sign of persistent asymptomatic mucosal inflammation predisposing to early recurrence. Indeed, it has been found that the clinical response usually precedes histological improvement and that lack of total histological resolution may be a predictor for relapse. ${ }^{3}$ Persistent high IMA flow probably reflects residual mucosal damage. This is supported by our findings of normalised PI in all patients with infectious colitis and nearly all patients with stable remission. In Crohn's disease, the postprandial SMA flow velocity correlates well 
with disease activity, ${ }^{36}$ and a persistently increased permeability despite clinical remission has been found to be associated with increased risk of relapse in Crohn's disease. ${ }^{37}$ Whether there is a similar mechanism in ulcerative colitis is not known. Therefore there may be a subset of patients without complete histopathological remission and increased vascular flow who are predisposed to early recurrent disease. It remains to be seen whether this new indicator is more helpful in treatment decisions, including prolonged steroid or immunosuppressive treatment in patients with remission but high mesenteric flow.

Several other new parameters for the assessment of disease activity have been proposed recently. Power Doppler sonography is a new method, allowing direct evaluation of blood flow resistance in the bowel wall, which correlates with inflammatory activity. ${ }^{38}$ Laboratory variables such as the leucocyte adhesiveness/ aggregation $^{39}$ and fibrin degradation products ${ }^{40}$ can be used to discriminate between patients with active and inactive disease. However, all of these have not yet been evaluated with respect to their predictive value.

In conclusion, our data show that the hyperdynamic splanchnic circulation in ulcerative colitis, as assessed by Doppler ultrasound, correlates with disease activity. Still more importantly, failure of the PI of the IMA to increase during clinical remission is highly indicative of early relapse.

1 Truelove SC, Witts LJ. Cortisone in ulcerative colitis. BMf 1955;ii:1041-8.

2 Saverymuttu SH, Hodgson HJG, Chadwick VS, et al. Differing acute phase responses in Crohn's disease and ulcerative colitis. Gut 1986;27:809-13.

3 Riley SA, Mani V, Goodman MJ, et al. Microscopic activity in ulcerative colitis: what does it mean? Gut 1991;32:1748 .

4 Gibson NR, Gibson PR, Donlan JD, et al. Modified Doppler flowmetry in the splanchnic circulation. Gastroenterology 1993;105:1029-34

5 Bolondi L, Gaiani S, Barbara L. Accuracy and reproducibility of portal flow measurement by Doppler US. F Hepatol 1991;13:269-73.

6 Sabba C, Weltin G, Cicchetti DV, et al. Observer variability in echo-Doppler measurements of portal flow in cirrhotic patients and normal volunteers. Gastroenterology 1990;98: 1603-11

7 De Vries PJ, Van Hattum J, Hoekstra JBL, et al. Duplex doppler measurements of portal venous flow in normal subjects. F Hepatol 1991;13:358-63.

8 Muller AF. Role of duplex Doppler ultrasound in the assessment of patients with postprandial abdominal pain. Gut 1992;33:460-5.

9 Ludwig D, Schwarting K, Korbel CM, et al. The postprandial portal flow is related to the severity of portal
hypertension and liver cirrhosis. F Hepatol $1998 ; 28: 631-8$.

10 Maconi G, Imbesi V, Porro IG. Doppler ultrasound measurement of intestinal blood flow in inflammatory bowel disease. Scand $\mathcal{F}$ Gastroenterol 1996;31:590-3.

11 Bolondi L, Gaiani S, Brignola C, et al. Changes in splanchnic hemodynamics in inflammatory bowel disease. Noninvasive assessment by Doppler ultrasound flowmetry. Scand F Gastroenterol 1992;27:501-7.

12 Sutherland LR, Martin F, Greer S, et al. 5-Aminosalicylate enema in the treatment of distal ulcerative colitis, proctosigmoiditis, and proctitis. Gastroenterology 1987;92: $1894-8$
13 Gosling RG, King DH, Newman DL, et al. Transcutaneous measurement of arterial blood velocity by ultrasound. In: Ultrasonics for industry conference papers. Guildford: IPC, 1969:16-32.

14 Moneta GL, Taylor DC, Helton S, et al. Duplex ultrasound measurement of postprandial intestinal blood flow: effect of meal composition. Gastroenterology 1988;95:1294-301.

15 McNeil BJ, Keeler E, Adelstein SJ. Primer on certain elements of medical decision making. $N$ Engl f Med 1975; 293:211-15.

16 Hulten L, Lindhagen J, Lundgren O, et al. Regional intestinal blood flow in ulcerative colitis and Crohn's disease. Gastroenterology 1977;72:388-96.

17 Brahme F, Lindström C. A comparative radiographic and pathological study of intestinal vaso-architecture in Crohn's disease and in ulcerative colitis. Gut 1970;11:92840.

18 Wakefield AJ, Sawyerr AM, Dhillon AP, et al. Pathogenesis of Crohn's disease: multifocal gastrointestinal infarction. of Crohn's disease: multif

19 Wakefield AJ, Pittilo RM, Sim R, et al. Evidence of persistent measles virus infection in Crohn's disease. $7 \mathrm{Med}$ Virol 1993;39:345-53

20 Sekizuka E, Grisham MB, Li $\mathrm{M}$, et al. Inflammationinduced intestinal hyperemia in the rat: role of neutrophils. Gastroenterology 1988;95:1528-34.

21 Brain SD, Williams TJ. Leucotrienes and inflammation. Pharmacol Ther 1990;46:57-66.

22 Knutson L, Ahrenstedt O, Odlind B, et al. The jejunal secretion of histamine is increased in active Crohn's disease. Gastroenterology 1990;98:849-54.

23 Levine JJ, Pettei MJ, Valderrama E, et al. Nitric oxide and inflammatory bowel disease: evidence for local intestinal production in children with active colonic disease. 7 Pediatr Gastroenterol Nutr 1998;26:34-8.

24 Kimura H, Hokari R, Miura S, et al. Increased expression of an inducible isoform of nitric oxide synthase and the formation of peroxynitrite in colonic mucosa of patients with active ulcerative colitis. Gut 1998;42:180-7.

25 Rees DC, Satsangi J, Cornelissen PL, et al. Are serum concentrations of nitric oxide metabolites useful for predicting centrations of nitric oxide metabolites useful for predicting
the clinical outcome of severe ulcerative colitis? Eur 7 Gastroenterol Hepatol 1995;7:227-30.

26 Guslandi M, Sorghi S, Polli D, et al. Rectal blood flow in ulcerative colitis. Am f Gastroenterol 1995;90:579-80.

27 Taylor GA. Blood flow in the superior mesenteric artery: estimation with Doppler US. Radiology 1990;174:15-16.

28 Sieber C, Beglinger C, Jaeger K, et al. Regulation of postprandial mesenteric blood flow in humans: evidence for a cholinergic nervous reflex. Gut 1991;32:361-6.

29 Gallavan RH, Chou CC, Kvietys P, et al. Regional blood flow during ingestion in the conscious dog. Am $\mathcal{F}$ Physiol 1980;238: $\mathrm{H} 220-5$.

30 Bendtsen F, Simonsen L, Henriksen JH. Effect on hemodynamics of a liquid meal alone and in combination with propranolol in cirrhosis. Gastroenterology 1992;102:1017-23.

31 Kelbaek H, Munck O, Christensen NJ, et al. Central haemodynamic changes after a meal. Br Heart $\mathscr{f}$ 1989;61: 506-9.

32 Powel-Tuck J, Day DW, Buckell NA, et al. Correlations between defined sigmoidoscopic appearances and other measures of disease activity in ulcerative colitis. Dig Dis Sci $1982 ; 27: 533-7$

33 Travis SPL, Farrant JM, Ricketts C, et al. Predicting outcome in severe ulcerative colitis. Gut 1996;38:905-10.

34 Langholz E, Munkholm P, Davidsen M, et al. Course of ulcerative colitis: analysis of changes in disease activity over years. Gastroenterology 1994;107:3-11

35 Moum B, Ekbom A, Vatn MH, et al. Clinical course during the 1st year after diagnosis in ulcerative colitis and Crohn's disease. Scand f Gatroenterol 1997;32:1005-12.

36 Ludwig D, Brüning A, Wiener S, et al. Postprandial splanchnic hemodynamics as activity index in inflammatory bowel disease (IBD): a prospective and controlled follow-up study [abstract]. Gastroenterology 1998;114: A392

37 Wyatt J, Vogelsang H, Hübl W, et al. Intestinal permeability and the prediction of relapse in Crohn's disease. Lancet 1993;341:1437-9.

38 Heyne R, Schreiber S, Wedel S, et al. Vascularisation and vascular resistance in the intestinal wall in inflammatory bowel disease as a sign for disease activity [abstract]. Gastroenterology 1998;114:A994.

39 Arber N, Berliner S, Hallak A, et al. Increased leucocyte adhesiveness/aggregation is a most useful indicator of disease activity in patients with inflammatory bowel disease. Gut 1995;37:77-80.

40 Kjeldsen J, Lassen JF, Brandslund I, et al. Markers of coagulation and fibrinolysis as measures of disease activity in inflammatory bowel disease. Scand f Gastroenterol 1998;33: $637-43$. 